\title{
Robust Region Tracking for Swarms via a Novel Utilization of Sliding Mode Control
}

\author{
Mark Bacon, Nejat Olgac, Rudy Cepeda-Gomez \\ Department of Mechanical Engineering, University of Connecticut, Storrs, USA \\ Email: olgac@engr.uconn.edu
}

Received December 23, 2011; revised January 23, 2012; accepted January 30, 2012

\begin{abstract}
Control of multi-agent autonomous swarms is studied for targeted flocking exercises. The desired decentralized control takes into account robustness against modeling uncertainties as well as bounded unknown forces. In this analysis, we consider the task of driving multiple agents to a moving "target region", as inter-agent repulsive forces help spread out the agents within the region. An unconventional form of sliding mode control is implemented to provide the robust attraction towards the region's center. For robustness a finite "boundary layer" is conceived, which corresponds to the desired target region. The flocking control forces are intentionally softened inside this target region, allowing agents to create a uniformly spaced formation guided by the inter-agent repulsion forces. Examples are given for moving circular and elliptical regions which illustrate the effectiveness of the proposed strategy.
\end{abstract}

Keywords: Multi-Agent Systems; Region Tracking; Sliding Mode Control

\section{Introduction}

Recently, decentralized control of multiple robotic agents has become an active area of research [1]. Mostly originating from biological inspiration [2-4], the mathematical modeling and control of these "swarms" have advanced to tackle a multitude of problems, such as flocking [5-10], formation flight [11], area coverage [12-15], and even hostile interactions with other swarms [16]. This paper discusses a hybrid procedure for flocking and area coverage.

The specific act of "flocking", where agents attempt to retain some proximity to their neighbors while aggregateing into a stable formation, has received significant attention. In 2003, Gazi and Passino proposed a first order model $[5,6]$, in which agents were driven to stable flocking behavior by biologically inspired momenta structures. In 2007, Yao et al. extended this concept to a second order model using a sliding mode controller [11]. The controller ensures that the agents' velocities follow the gradient descent of some desired momenta profiles.

Olfati-Saber handled flocking for a second order model [7] where each agent's motion is determined by artificial potential energy components. A virtual leader is introduced to prevent fragmentation into smaller groups by creating a common attractive target for all agents. The assumption of universal knowledge of the virtual leader by all the agents is shown to be unnecessary in 2009 by Su et al. [8].
Tanner et al. [9] demonstrates stability of a swarm with no leader for arbitrarily quickly switching network topologies, provided that the swarm remains connected. Zavlanos and Tanner [10] enforce the connectivity of the swarm through a hybrid control. This controller uses local estimates of the network topologies.

Controlled distribution of agents over a wide area (called "coverage control") is studied by Cortes et al. [12-14]. The majority of these approaches analyze static convex regions. The motion of the agents is determined using gradient descent of some artificial potential.

A hybrid of "flocking" and "area coverage" control of agents inside a moving region is studied in Cheah [15] again using some artificial potential fields. The potential fields are described point wise in the space of the motion, including the inter-agent forces. Our work attempts to solve a similar problem, flocking and area coverage within a target region, but using a completely different approach. We extend the traditional sliding mode controller (SMC) [17] and introduce a new boundary layer concept to achieve the task. This controller competes against modeling uncertainties and bounded unknown forcing functions. The SMC robustly draws all agents towards the target region's center. However when the agents are inside the region the control is softened allowing the inter-agent repulsive forces to determine the spacing between agents. The region's perimeter is shown to be upheld successfully by properly selecting the control gains. 
Classically, SMC is used for robustizing the control within a desirably small boundary layer [18]. Our novelty lies in performing SMC with a relatively large boundary layer which corresponds to the target region. When the steady state occurs, the swarm will be entrapped within that region. Another critical departure from the traditional SMC is at the deployment of the boundary layer. As described in the text, this leads to a new and desirable feature: sliding occurs at the same time in all spatial dimensions, instead of separate instants.

The resulting decentralized control guides the agents to achieve area coverage within the moving target region. The approach to the target by the agents is asymptotic, and collisions are avoided. Discussions on stability of the controlled dynamics, as well as the disturbance rejection capabilities are included.

The paper is organized as follows: Section 2 covers the governing dynamics of the system and outlines the specific objective. The third section develops the SMC and illustrates its robustness properties for a circular target region. We also provide an analysis of the pessimistic upper bounds for the inter-agent repulsive forces. The effectiveness of such a controller is demonstrated in Section 4. Section 5 expands the analysis to elliptical regions, and the results of this expansion are presented in Section 6. Finally, conclusions are given in Section 7. As a common notation within the text, we denote vectors and matrices with a boldface font, and scalars with italics.

\section{System Dynamics and Problem Formulation}

In this paper we consider an $M$-agent swarm in a 2-D environment. Each agent's dynamics are governed by the following equation

$$
m_{i} \ddot{\mathbf{x}}_{i}+b_{i} \dot{\mathbf{x}}_{i}=\mathbf{u}_{i}+\mathbf{f}_{i}^{r}\left(Y_{i}\right)+\mathbf{f}_{i}^{u} \in \mathfrak{R}^{2} \quad i=1,2, \cdots M
$$

where $\mathbf{x}_{i} \in \mathfrak{R}^{2}$ is the position vector of the $i^{\text {th }}$ agent. $m_{i}$ and $b_{i}$ are the mass and drag coefficients of that agent. They are assumed to be uncertain modeling parameters with nominal values, $\bar{m}$ and $\bar{b}$, and bounded uncertainties,

$$
\begin{aligned}
& m_{i}=\bar{m}+\tilde{m}_{i},\left|\tilde{m}_{i}\right| \leq \Delta m \ll \bar{m}, \\
& b_{i}=\bar{b}+\tilde{b}_{i},\left|\tilde{b}_{i}\right| \leq \Delta b \ll \bar{b} \quad i=1,2, \cdots, M
\end{aligned}
$$

$\mathbf{u}_{i}$ is the control force on the $i$-th agent and $\mathbf{f}_{i}^{r}\left(Y_{i}\right)$ is the inter-agent repulsion force which is unknown to the controller, except its conservative upperbound $\left\|\mathbf{f}_{i}^{r}\right\| \leq f_{\max }^{r}$. These forces are directly linked to the geometric distribution of the agents at any given moment. A focused effort on the formation of these forces and determination of their upperbounds are presented later in the text. The set $Y_{i}$ contains the positions of neighbors of agent $i$, defined by $Y_{i}=\left\{\mathbf{x}_{j}:\left\|\mathbf{x}_{i}-\mathbf{x}_{j}\right\| \leq r_{Y}, i \neq j\right\}$, where $r_{Y}$ is the radius of the neighborhood. We call it the radius of interaction. Only those agents that are in $Y_{i}$, influence the dynamics of agent $i$. The $\mathbf{f}_{i}^{u}$ term in (1) represents a friction-like unknown force (indicated by the superscript " $u$ ") which opposes the motion. It is assumed to be smoothly varying and upperbounded, $\left\|\mathbf{f}_{i}^{u}\right\| \leq f_{\max }^{u}$.

The objective of the control is to drive all agents from a set of arbitrary initial conditions to within a moving circular target region. This region is defined by

$\left\|\mathbf{x}-\mathbf{x}_{d}\right\| \leq r_{\text {circ }}$, of which $\mathbf{x}_{d}(t) \in \mathfrak{R}^{2}$ is the center and $r_{\text {circ }}$ is the radius. The control should be robust against modeling uncertainties (the mass and the drag constants) as well as the uncertain repulsion and disturbance forces. Initially, we will consider the target region to be a circle, and later the concept will be extended to an ellipse.

\section{Inter-Agent Repulsive Force}

The intent of the repulsive forces is to create "personal space" for each agent $i$, as well as to avoid collision of the agents, by pushing the neighbors away. The resultant of such inter-agent forces on agent $i$ is denoted by $\mathbf{f}_{i}^{r}$. These forces come from those agents within the neighborhood of $i$ and they meet the following criteria:

1) The force is continuous along $\left\|\mathbf{x}_{i}-\mathbf{x}_{j}\right\| \in\left(0, r_{Y}\right]$ and attains its maximum at $\left\|\mathbf{x}_{i}-\mathbf{x}_{j}\right\|=0$.

2) They diminish at the edge of the neighborhood: $\left.\mathbf{f}_{i}^{r}\right|_{\left\|\mathbf{x}_{i}-\mathbf{x}_{j}\right\|=r_{Y}}=0$.

In this study, we take the formation of these forces as

$$
\mathbf{f}_{i}^{r}=\sum_{\mathbf{x}_{j} \in Y_{i}}\left(r_{Y}-\left\|\mathbf{x}_{i}-\mathbf{x}_{j}\right\|\right)^{2} \frac{\mathbf{x}_{i}-\mathbf{x}_{j}}{\left\|\mathbf{x}_{i}-\mathbf{x}_{j}\right\|}
$$

They are, however, unknown to the agents except their pessimistic aggregate upperbound. As such, in the control logic they are treated as part of the bounded uncertainty.

\section{Sliding Mode Controller (SMC)}

The objective of the control is to bring the agents to within the target region, which is taken as a circle for the first part of the paper. Following the traditional SMC formulations [17-19] one starts with the definition of error to be minimized,

$$
\mathbf{e}_{i}=\mathbf{x}_{d}-\mathbf{x}_{i} \in \mathfrak{R}^{2}
$$

which is the vector connecting an individual agent to the center $\mathbf{x}_{d}$ of the target region. The sliding function is then defined as a Hurwitz combination of the error

$$
\mathbf{s}_{i}=\lambda \mathbf{e}_{i}+\dot{\mathbf{e}}_{i} \in \mathfrak{R}^{2}
$$

The sliding mode controller first reduces $\left\|\mathbf{s}_{i}\right\|$ during 
the "approach phase", then it maintains $\left\|\mathbf{s}_{i}\right\|$ within a confinement in the pursuant "sliding phase". In both phases we utilize LaSalle's theorem [20], to enforce the attracttivity to this confinement. A positive definite Lyapunov candidate for agent $i$ is proposed as

$$
V_{i}=\frac{1}{2} \mathbf{s}_{i}^{T} \mathbf{s}_{i}>0
$$

of which the derivative is forced to be negative

$$
\dot{V}_{i}=\mathbf{s}_{i}^{T} \dot{\mathbf{s}}_{i}>0
$$

Combining (1), (4) and (5), results in

$$
\dot{\mathbf{s}}_{i}=\lambda \dot{\mathbf{e}}_{i}+\ddot{\mathbf{x}}_{d}-\frac{1}{m_{i}}\left(\mathbf{u}_{i}+\mathbf{f}_{i}^{r}+\mathbf{f}_{i}^{u}-b_{i} \dot{\mathbf{x}}_{i}\right)
$$

The control, $\mathbf{u}_{i}$, is selected such that the $\mathbf{s}_{i}$ dynamoics behave according to $\dot{\mathbf{s}}_{i}=-K_{1} \cdot \mathbf{s}_{i}-K_{2} \frac{\mathbf{s}_{i}}{\left\|\mathbf{s}_{i}\right\|}$, fulfilling the condition in (7).

The proposed $\mathbf{s}_{i}$ dynamics, ignoring the uncertainties can be achieved with the deployment of a control as

$$
\mathbf{u}_{i}=m_{i} K_{1} \mathbf{s}_{i}+m_{i}\left[\lambda \dot{\mathbf{e}}_{i}+\ddot{\mathbf{x}}_{d}\right]+b_{i} \dot{\mathbf{x}}_{i}+m_{i} K_{2} \frac{\mathbf{s}_{i}}{\left\|\mathbf{s}_{i}\right\|}
$$

Substituting (9) into (8), but including the uncertain terms

$$
\dot{\mathbf{s}}_{i}=-K_{1} \mathbf{s}_{i}+\left(-K_{2} \frac{\mathbf{s}_{i}}{\left\|\mathbf{s}_{i}\right\|}-\mathbf{f}_{i}^{r}-\mathbf{f}_{i}^{u}\right)
$$

Considering the worst case contributions of the uncertainties;

$$
\dot{\mathbf{s}}_{i, \text { worst case }}=-K_{1} \mathbf{s}_{i}+\left(-K_{2}+f_{\max }^{r}+f_{\max }^{u}\right) \frac{\mathbf{s}_{i}}{\left\|\mathbf{s}_{i}\right\|}
$$

Selecting $K_{2}=f_{\max }^{r}+f_{\max }^{u}$ makes $\dot{\mathbf{s}}_{i \text {,worst case }}=-K_{1} \mathbf{s}_{i}$, which forces $\dot{V}_{i}<0$ at all times. Notice that $\mathbf{s}_{i} \in \mathfrak{R}^{2}$ is a vector. For small $\left\|\mathbf{s}_{i}\right\|$ values, the $\mathbf{s}_{i} /\left\|\mathbf{s}_{i}\right\|$ term in the control (9) generally brings undesirable control chatter, i.e., small departures of $\left\|\mathbf{s}_{i}\right\|$ from zero may result in large swings in $\mathbf{u}_{i}$. These swings (chatter) can be alleviated using a saturation function approximation [17-19] within a boundary layer $\left\|\mathbf{s}_{i}\right\| \leq \varepsilon$

$$
\operatorname{sat}\left(\mathbf{s}_{i}, \varepsilon\right)=\left\{\begin{array}{cc}
1 & \text { for }\left\|\mathbf{s}_{i}\right\|>\varepsilon \\
\left\|\mathbf{s}_{i}\right\| / \varepsilon & \text { for }\left\|\mathbf{s}_{i}\right\| \leq \varepsilon
\end{array} \quad \text { with } \varepsilon=\lambda r_{\text {circ }}\right.
$$

The treatment up to this point is conventional except the large boundary layer expression $\varepsilon$. The system's behavior is robust outside this boundary layer, and the controller (9) is designed to drive the system towards $\left\|\mathbf{s}_{i}\right\| \leq \varepsilon$. Notice the selection of (12) for $\varepsilon$, at the steady state (i.e. $\dot{\mathbf{e}}_{i} \approx 0$ ), the error $\mathbf{e}_{i}$ remains bounded within

$$
\left\|\mathbf{x}_{i}-\mathbf{x}_{d}\right\|=\left\|\mathbf{e}_{i}\right\| \leq \frac{\varepsilon}{\lambda}=r_{\text {circ }}
$$

This constitutes the critical departure from the convention and the essence of the novel contribution in this pa- per. $\varepsilon$ is not a small boundary width, but large, and it compares to the target region $r_{\text {circ }}$. Robust controller (9) is softened by the saturation function (12) replacing $\mathbf{s}_{i} /\left\|\mathbf{s}_{i}\right\|$. The objective of this softening is to let the inter-agent repulsion forces, $\mathbf{f}_{i}^{r}$, take charge in the distribution of the agents within the target region. Notice that the boundary layer defined in (12) is circular in $\left(s_{1}, s_{2}\right)$ space, and so is the respective $\left(x_{1}, x_{2}\right)$ counterpart, as per (13). This feature points us to circular target regions most naturally.

In summary, our implementation of the boundary layer is novel and substantially different from the traditional usage. The concept is usurped here to enforce a desired "target state" as opposed to "control chatter abatement". Thus, $\varepsilon$ is selected as a finite quantity here, by definition, as opposed to a small number in the conventional deployment, where the size of the boundary layer also influences the control chatter cut-off frequency. We bring a novel philosophy by using the "boundary layer" concept to bound the final distribution of the agents. This provides not only the intended chatter abatement $[18,19]$, but also softens the attraction of the target region's center. Outside this region, the robustizing term is in full effect and drives the agents towards the region. Inside the region however, it is tolerant towards the inter-agent spacing forces (i.e., repulsion). The control expression in (9) becomes

$$
\mathbf{u}_{i}=\bar{b} \dot{\mathbf{x}}_{i}+\bar{m}\left[\lambda \dot{\mathbf{e}}_{i}+\ddot{\mathbf{x}}_{d}+K_{1} \cdot \mathbf{s}_{i}+K_{2} \cdot \operatorname{sat}\left(\mathbf{s}_{i}, \varepsilon\right) \frac{\mathbf{s}_{i}}{\left\|\mathbf{s}_{i}\right\|}\right]
$$

where the known nominal values of $m_{i}$ and $b_{i}$ are also included. Substituting (14) into (8), the $\mathbf{s}_{i}$-dynamics become

$$
\begin{aligned}
\dot{\mathbf{s}}_{i}= & \frac{1}{m_{i}}\left[\tilde{m}_{i}\left(\lambda \dot{\mathbf{e}}_{i}+\ddot{\mathbf{x}}_{d}\right)+\tilde{b}_{i} \dot{\mathbf{x}}_{i}\right. \\
& \left.-\bar{m}\left(K_{1} \cdot \mathbf{s}_{i}+K_{2} \cdot \operatorname{sat}\left(\mathbf{s}_{i}, \varepsilon\right) \frac{\mathbf{s}_{i}}{\left\|\mathbf{s}_{i}\right\|}\right)-\mathbf{f}_{i}^{r}-\mathbf{f}_{i}^{u}\right]
\end{aligned}
$$

1) When $\left\|\mathbf{s}_{i}\right\| \gg \varepsilon$, the dynamics is in the approach phase, $\operatorname{sat}\left(\mathbf{s}_{i}, \varepsilon\right)=1$. The agents are likely to be distant from each other, as per the definition of $\mathbf{s}_{i}$ given in (5), therefore the repulsive forces are expected to be small. The derivative of the Lyapunov function is

$$
\begin{array}{r}
\dot{V}_{i}=\frac{1}{m_{i}} \mathbf{s}_{i}^{T}\left[\tilde{m}_{i}\left(\lambda \dot{\mathbf{e}}_{i}+\ddot{\mathbf{x}}_{d}\right)+\tilde{b}_{i}\left(\dot{\mathbf{x}}_{i}\right)\right. \\
\left.-\bar{m}\left(K_{1} \cdot \mathbf{s}_{i}+K_{2} \frac{\mathbf{s}_{i}}{\left\|\mathbf{s}_{i}\right\|}\right)-\mathbf{f}_{i}^{u}\right]
\end{array}
$$

In order to ensure $\dot{V}_{i}<0$, we select

$$
K_{2} \geq \frac{1}{\bar{m}}\left(f_{\max }^{u}+\Delta m\left\|\lambda \dot{\mathbf{e}}_{i}+\ddot{\mathbf{x}}_{d}\right\|+\Delta b\left\|\dot{\mathbf{x}}_{i}\right\|\right)
$$


which guarantees the attractivity of the boundary region $\left\|\mathbf{s}_{i}\right\|=\varepsilon$.

2) When $\left\|\mathbf{s}_{i}\right\| \leq \varepsilon$, sat $\left(\mathbf{s}_{i}, \varepsilon\right)=\left\|\mathbf{s}_{i}\right\| / \varepsilon$, the agents are more compactly positioned and the $\mathbf{f}_{i}^{u}$ term grows to be more pronounced. Equations (7) and (15) yield

$$
\begin{aligned}
\dot{V}_{i} & =\frac{1}{m_{i}} \mathbf{s}_{i}^{T}\left[\tilde{m}\left(\lambda \dot{\mathbf{e}}_{i}+\ddot{\mathbf{x}}_{d}\right)+\tilde{b} \dot{\mathbf{x}}_{i}\right. \\
& \left.-\bar{m}\left(K_{1} \cdot \mathbf{s}_{i}+K_{2} \cdot \frac{\mathbf{s}_{i}}{\varepsilon}\right)-\mathbf{f}_{i}^{r}-\mathbf{f}_{i}^{u}\right]
\end{aligned}
$$

to enforce $\dot{V}_{i}<0$, at the border of the boundary layer, that is, where $\operatorname{sat}\left(\mathbf{s}_{i}, \varepsilon\right)=1$, we consider the most pessimistic case for uncertainties and select the robustizing gain as

$$
K_{2} \geq \frac{1}{\bar{m}}\left(f_{\max }^{r}+f_{\max }^{u}+\Delta m\left\|\lambda \dot{\mathbf{e}}_{i}+\ddot{\mathbf{x}}_{d}\right\|+\Delta b\left\|\dot{\mathbf{x}}_{i}\right\|\right)
$$

This yields the attractivity of the swarm to within the target circle. Once within the boundary layer, the controlled dynamics would comply with

$$
\begin{aligned}
& \dot{\mathbf{s}}_{i}+\frac{\bar{m}}{m_{i}}\left(K_{1}+\frac{K_{2}}{\varepsilon}\right) \mathbf{s}_{i} \\
& =\frac{1}{m_{i}}\left[-\mathbf{f}_{i}^{r}-\mathbf{f}_{i}^{u}+\tilde{b}_{i} \dot{\mathbf{x}}_{i}+\tilde{m}_{i}\left[\lambda\left(\dot{\mathbf{x}}_{d}-\dot{\mathbf{x}}_{i}\right)+\ddot{\mathbf{x}}_{d}\right]\right]=\psi
\end{aligned}
$$

Equation (20) represents a low pass filter against the perturbation, $\psi$ (self evident from the previous equation) which entails the uncertainties. This filter attenuates high frequency components of the $\mathbf{s}_{i}$ dynamics emanating from perturbations with a cutoff frequency at

$$
\omega_{i}=\frac{\bar{m}}{m_{i}}\left(K_{1}+\frac{K_{2}}{\varepsilon}\right)
$$

This strategy is borrowed from [19], which also contains experimental validation of the concept. Entrapment of the agents within the boundary layer makes the robustizing part of the controller, $K_{2} \mathbf{s}_{i} / \varepsilon$, less effective. Then, the agent distributions are primarily influenced by $\mathbf{f}_{i}^{r}$, as well as $\mathbf{f}_{i}^{u}$ (which is small as velocities are expected to be small). This yields the desired "area capture".

Notice that (19) suggests a more conservative feedback gain, $K_{2}$, than (17). In order to maintain continuity at the $\left\|\mathbf{s}_{i}\right\|=\varepsilon$ boundary, we use (19) throughout.

\section{Evaluation of the Repulsive Force Bound}

The upper-bound, $f_{\max }^{r}$, used in (19) represents the largest resultant force exerted on an agent due to the interagent repulsions and it is assumed known a priori. We present here a numerical procedure to assess that value. When the agents are forced within the target circle, they are expected to space out in a nearly uniform manner. Consequently, those agents at the periphery would be exposed to larger net repulsion forces than those inside. To estimate an extremum for these forces, i.e., $f_{\max }^{r}$, we create a model distribution of uniformly spaced agents within the circular target region. This formation is created by positioning the agents over nested circles with roughly uniform spacing (i.e., $\delta_{1} \approx \delta_{2} \approx \delta_{r}$ in Figure 1 for 30 agents.). We then numerically determine the resultant repulsion forces, using (3), on agents at the periphery (e.g., A in Figure 2) due to agents in the neighborhood (shaded in the figure). Considering isotropic and uniform distribution of agents within a circle, all peripheral agents should be exposed to similar calculated $f_{\max }^{r}$ values. Target geometries other than a circle would bring anisotropic $f_{\max }^{r}$ analysis. This point alone confines this scheme to circular targets. We will show the complications even for the elliptical case in latter sections.

\section{Case Studies for Circular Targets}

In order to demonstrate the effectiveness of the proposed control strategy, we present some case studies. The parameters in Table $\mathbf{1}$ are common to all cases considered. The circular target is again defined by its center, $\mathbf{x}_{d}(t) \in \mathfrak{R}^{2}$, and radius, $r_{\text {circ }}$.

Case study 1 is on a group of 30 agents aggregating within a non-moving circular region with $r_{\text {circ }}=2$, using the aforementioned evaluation of $f_{\max }^{r}$. The parameters

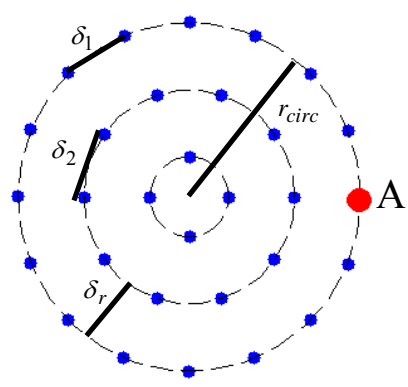

Figure 1. Model distribution of 30 agents.

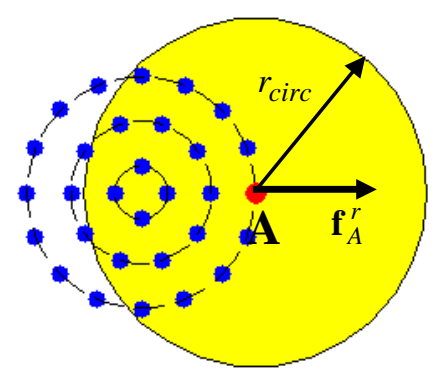

Figure 2. Neighborhood of agent a.

Table 1. Simulation parameters.

\begin{tabular}{ccc}
\hline $\bar{m}=1$ & $\Delta m=0.05$ & $r_{Y}=3$ \\
\hline $\bar{b}=1$ & $\Delta b=0.05$ & \\
$\lambda=1$ & $K_{1}=5$ & \\
\hline
\end{tabular}


$m_{i}$ and $b_{i}$ are fixed but randomly selected based on a uniform probability distribution within the known bounds of uncertainty ( $\Delta m$ and $\Delta b$ ). We also consider an unknown, time-varying friction-like force,

$\mathbf{f}_{i}^{u} \equiv-(20+5 \sin (2 t)) \dot{\mathbf{x}}_{i} /\left\|\dot{\mathbf{x}}_{i}\right\|$ which is only known to the controller by its upperbound $f_{\max }^{u}=25$.

Figure 3 shows the time-lapsed frames of the dynamoics. The agents inside the region remain almost evenly distributed, which indicates that our prediction of supremum of repulsion forces, $f_{\max }^{r}$, is appropriate. The first two frames do not have as many agents due to their selected remote starting positions.

We introduce a numerical metric for a quantitative
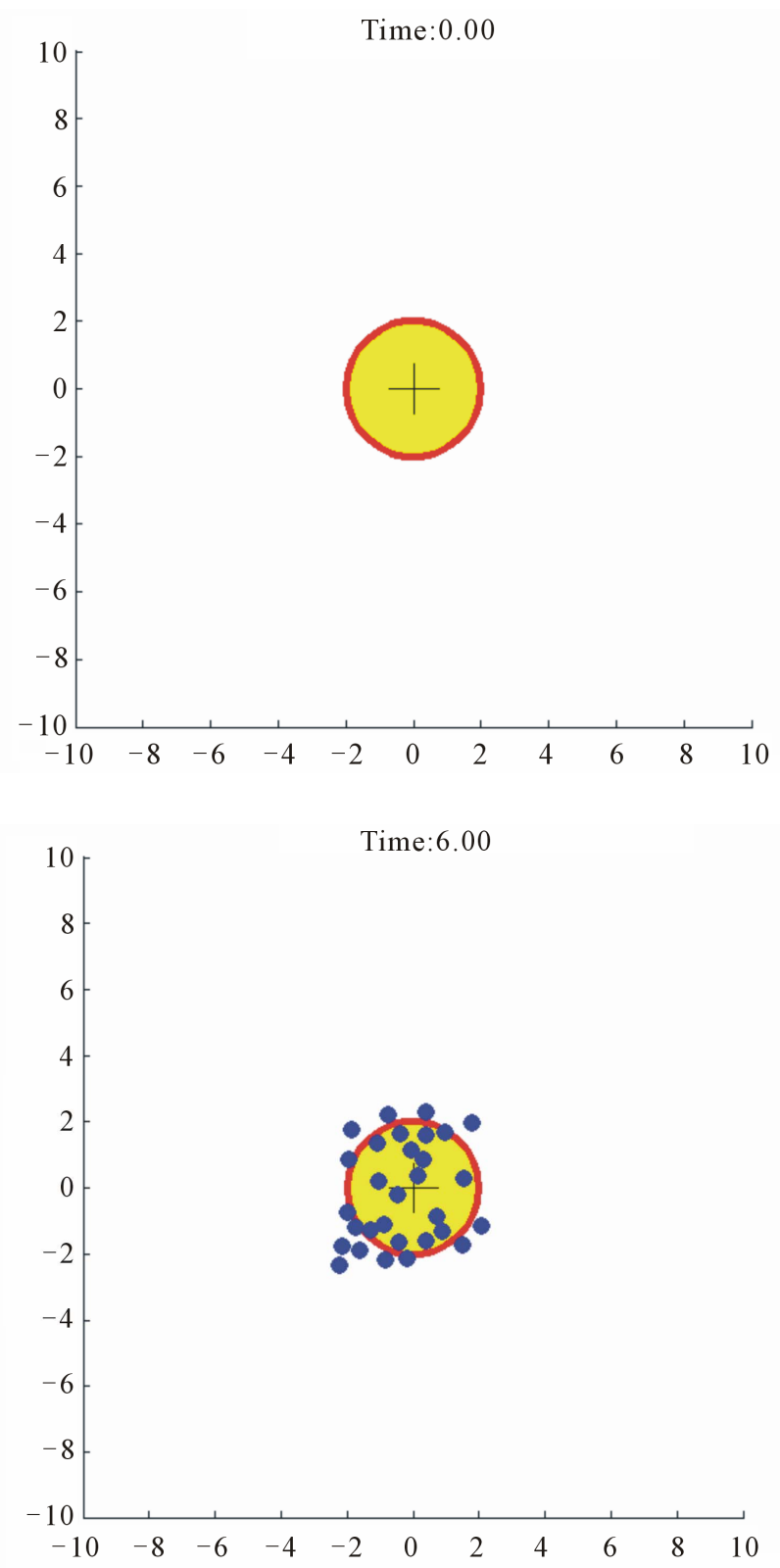

comparison among various agent distributions vis-à-vis the target region to be occupied. It is called the coverage index and defined by:

$$
C=\overline{r_{\min }} / \sqrt{A / M}
$$

where $\overline{r_{\min }}$ is the average of the minimum distances of each agent

$$
\overline{r_{\min }}=\sum_{i=1}^{M} r_{i, \min } / M
$$

$r_{i, \min }$ is the distance of agent $i$ to its nearest neighbor; $A$ is the area of the target region, and $M$ is the number of agents. This dimensionless quantity, $C$, is close to 1 for a uniformly spaced distribution within the target region. $C$
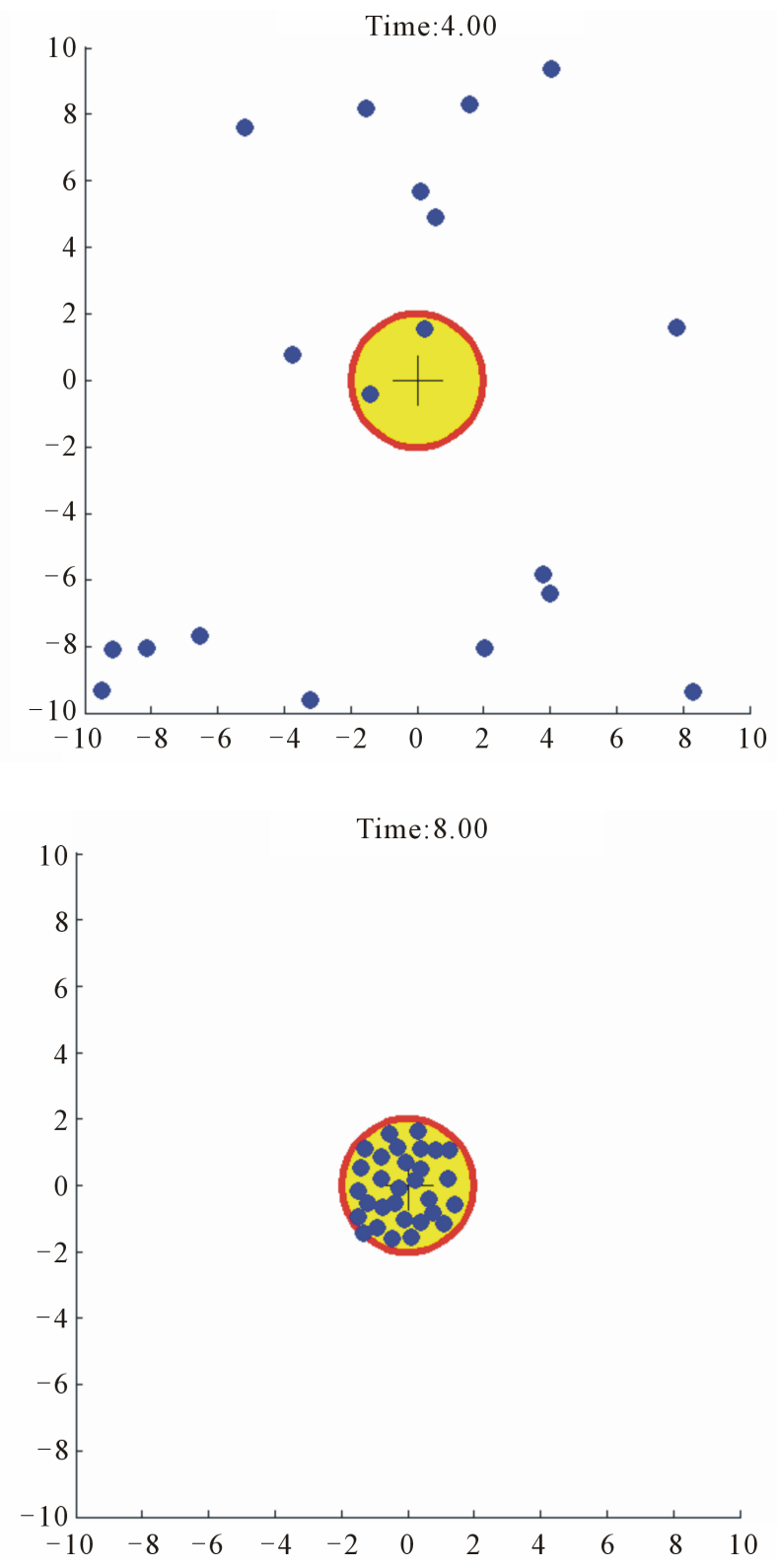

Figure 3. Case study 1: 30 agents driven to a fixed target circle. 
$>1$ and $C<1$ would imply dispersion outside the target region and bunching up within the target region cases respectively.

The variation of $C$ in time is shown in Figure 4 for this case study. The values marked correspond to the 4,6 and $8 \mathrm{sec}$. snapshots depicted in Figure 3. The measure, $C$, of coverage fully describes the slightly bunched coverage in the last frame (i.e., $8 \mathrm{sec}$.), $C \approx 0.7$ simply indicates that the determination of $f_{\max }^{r}$ was over conservative.

Case study 2 (Figure 5) shows 60 agents tracking a moving circular region of radius 2 , the center of which is moving according to $\mathbf{x}_{d}=[3 \sin (4 t) 3 \cos (2 t)]^{T}$ which is shown as a trace in Figure 5. All of the agents again aggregate inside the region despite the parameter uncertainties, upper-bounded unknown forces, and inter-agent repulsion forces.

Following the quantitative discussion on Case study 1 we present the coverage index variations for this example (see Figure 6). Again, the corresponding points of 4, 5, 6 and 10 sec. snapshots in Figure 5 are displayed in this figure.

One can notice that $C \approx 0.93$ declares a uniform and desirable filling of the circular target at $10 \mathrm{sec}$. This is achieved despite the uncertainties in the dynamics and moving target region, which shows a very effective control.

Time traces of $\mathbf{s}_{i} \in \mathfrak{R}^{2}$ are shown in Figure 7. The agent enters the sliding phase within 0.8 seconds, which roughly corresponds to 4 times the time constant

$1 / K_{1}=0.2$ seconds of (15) starting from large values of $\mathbf{s}_{i}$. Note the sliding manifold of $\left\|\mathbf{s}_{i}\right\|<2$ is unnoticeably small in the figure.

Figure 8 shows the control force, repulsive forces and the uncertain force on the same agent. The resultant of all forces on the agent at the steady state is periodic in nature, corresponding to the motion of the moving region.

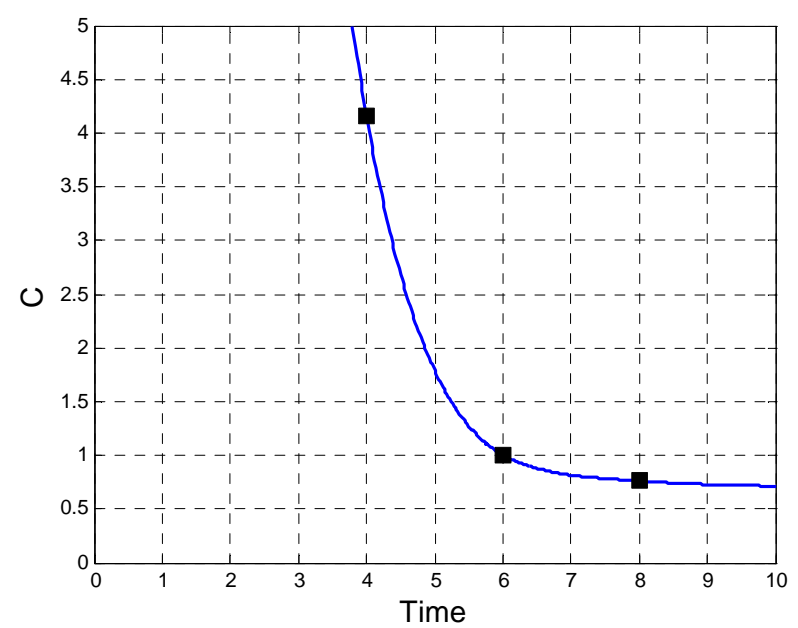

Figure 4. Variation of the coverage index $C$ in Case study 1.
Expected from the trajectories, the dominant periods are $\pi / 2$ and $\pi$ sec., in $x_{1}$ and $x_{2}$ direction, respectively. The general features of Figure 8 remain the same for all the agents with some small numerical variations. Another point of interest is that the oscillatory behavior starts at about $0.8 \mathrm{sec}$., which corresponds to the initiation of sliding and the entrapment of the agents within the target circle.

\section{Analysis for Elliptical Targets}

The ability to manage a swarm in different shapes has many applications, such as squeezing agents through a narrow strait. As a natural extension from the circular distribution previously presented, we expand the analysis to an elliptical flocking. Cheah et al. [15] also treat this type of target region, as mentioned, using potential functions for control. The approach is completely different in this paper. While we study the operation in $2-\mathrm{D}$, the concepts can be easily extended for an n-D system.

We define the target region, by its center $\mathbf{x}_{d}(t) \in \mathfrak{R}^{2}$, and the oblique ellipse by $\left(\mathbf{x}-\mathbf{x}_{d}\right)^{T} \mathbf{T}\left(\mathbf{x}-\mathbf{x}_{d}\right)=1$, where $\mathbf{T} \in \mathfrak{R}^{2 \times 2}$ is a symmetric matrix. This matrix contains information on the scaling factors of major and minor axes (from here on denoted by $r_{1}$ and $r_{2}$ ), as well as the angle of the major axis $\phi$. In the interest of space we depict these features in a later figure.

\subsection{Modifications to the Controller}

We again define a boundary layer, but this time as an elliptical region such that it becomes the target ellipse. We adopt a new vector norm for this process

$$
\|\mathbf{y}\|_{T}=\sqrt{\mathbf{y}^{T} \mathbf{T y}}
$$

where the subscript $T$ stands for transformed state. Then the development of Section 3 is repeated, to deploy the boundary layer concept. The goal is to ensure robust attraction towards the boundary layer (i.e., the target region) $\left\|\mathbf{s}_{i}\right\|_{T} \leq \varepsilon$. That means at the steady state, when the dynamics settle, we expect

$$
\left\|\mathbf{e}_{i}\right\|_{T} \leq \frac{\varepsilon}{\lambda}=1
$$

which implies an entrapment within the elliptical target. In order to achieve this, we use the same robust control logic as in Equation (14), except the new definition of $\operatorname{sat}(\mathbf{y}, \varepsilon)$ as

$$
\operatorname{sat}(\mathbf{y}, \varepsilon)=\left\{\begin{array}{cc}
1 & \text { for }\|\mathbf{y}\|_{T}>\varepsilon \\
\|\mathbf{y}\|_{T} / \varepsilon & \text { for }\|\mathbf{y}\|_{T} \leq \varepsilon
\end{array}\right.
$$

Notice that in (14) the robustizing force with $K_{2}$ is still acting in the same sense as before, i.e., assisting $\mathbf{s}_{i}^{T} \dot{\mathbf{s}}_{i}<0$. In summary, introducing the norm definition of (24) the circular target can be converted into an ellip- 

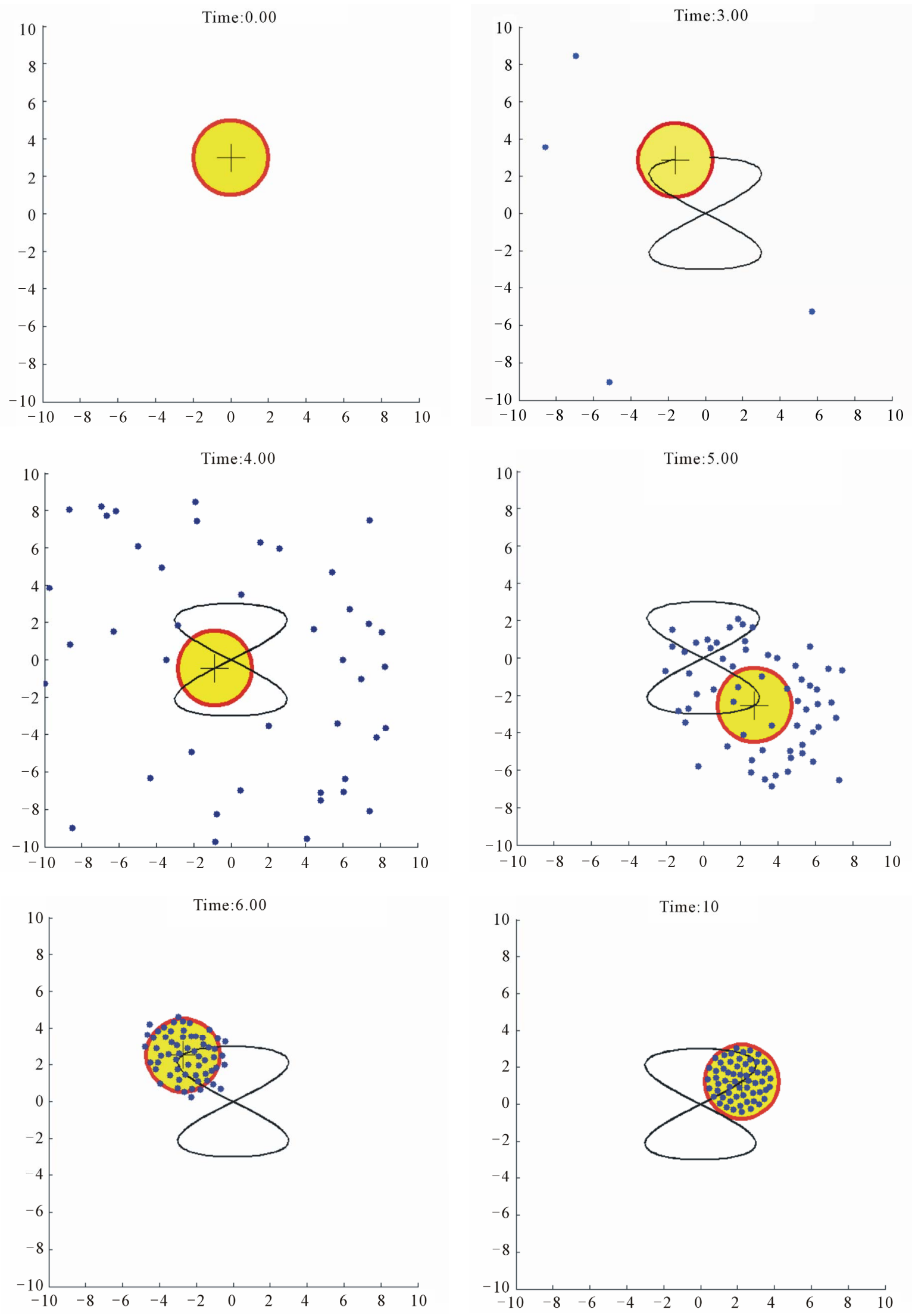

Figure 5. Case study 2: 60 agents tracking a moving circular region. 


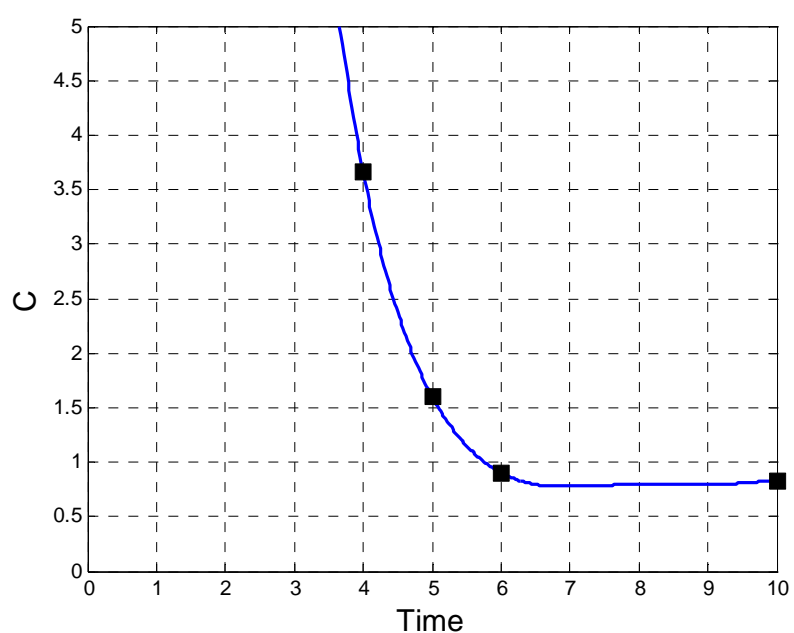

Figure 6. Variation of the coverage index $C$ in Case study 2.
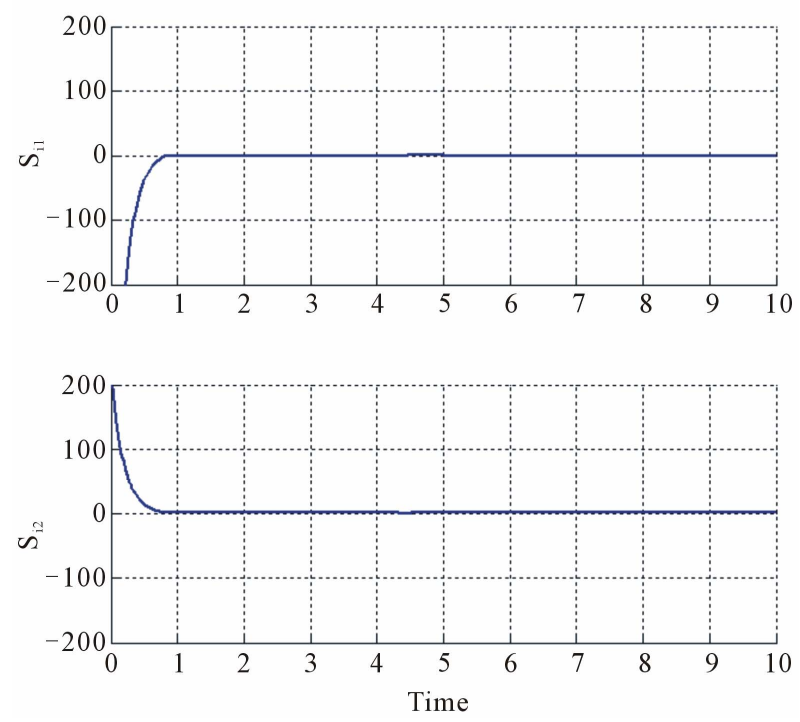

Figure 7. Sliding functions of an agent from Case study 2.

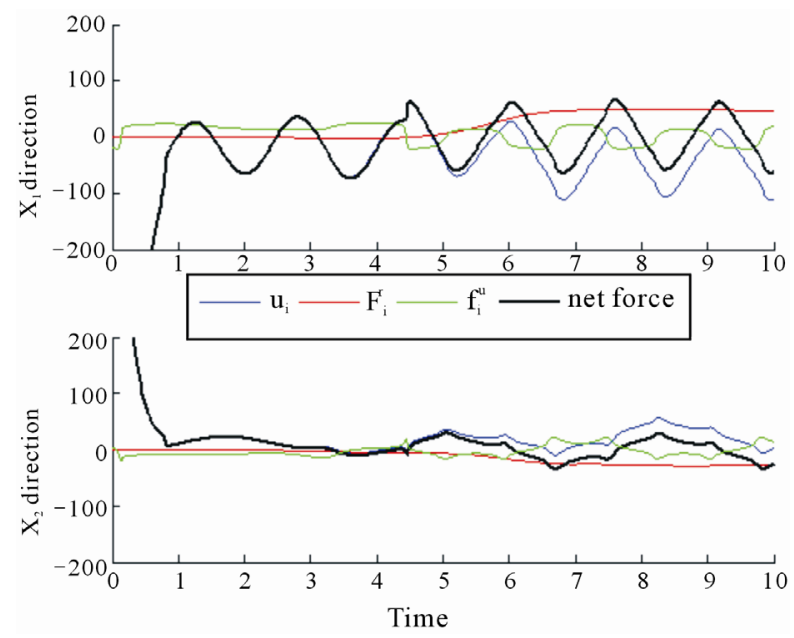

Figure 8. Forces exerted on an agent from Case Study 2. tical one. The remainder of the control operation stays the same, except the determination of $f_{\max }^{r}$.

\subsection{Estimating the Upperbound of the Repulsion Force for an Elliptical Configuration}

As proposed in Section 4, $f_{\max }^{r}$ quantity is needed as the a priori knowledge in the control. A conservative upperbound for this quantity can be obtained if we consider bunching of the agents within a circle of radius $r_{2}$, the minor axis radius, (Figure 9), instead of evenly distributing them inside the target ellipse. We evaluate the repulsive forces in this distribution using the similar arguments as in Figures $\mathbf{1}$ and $\mathbf{2}$.

In an elliptical distribution, however, the directional isotropy of the maximum repulsive forces is lost. Therefore we divide the domain into 4 separate zones (Figure 10) during the approach phase. These areas are determined by the aspect ratio of the target ellipse, and are used to schedule the gains, compensating for the lack of isotropy. Figure 11 shows how the repulsive forces vary when agents are distributed evenly within the entire elliptical region (with $r_{1}=4$ and $r_{2}=2$ ). Typically, the outside agents (i.e., those which are on the periphery) receive the highest repulsion forces. Furthermore, the

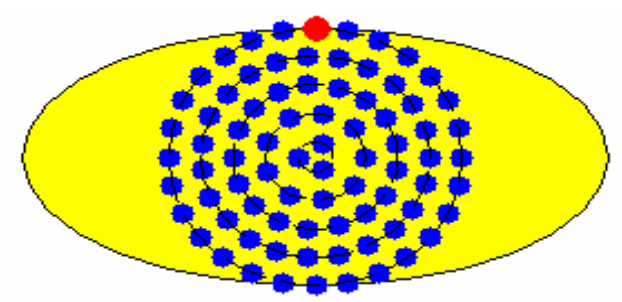

Figure 9. 80 agents in a worst-case distribution inside the target ellipse.

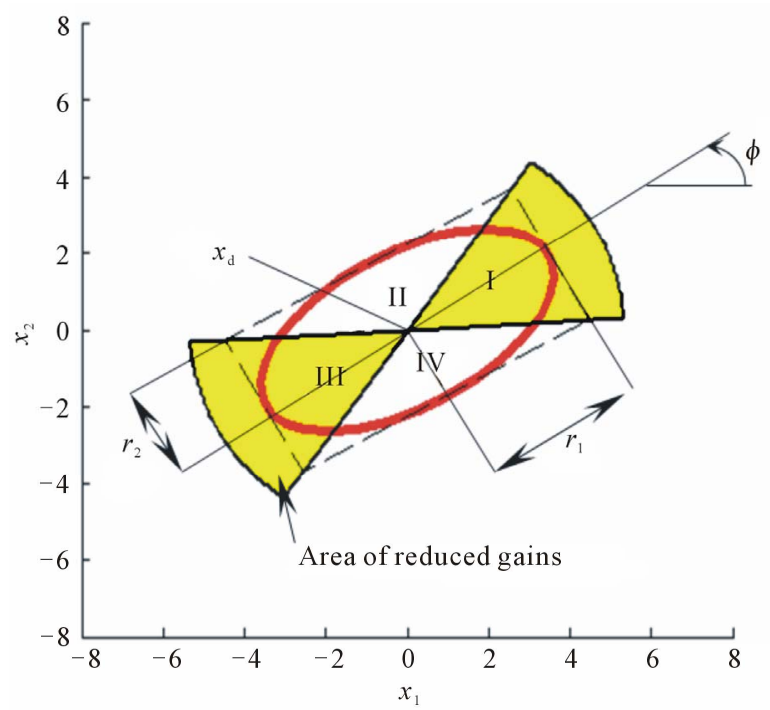

Figure 10. Areas of scheduled control gains. 


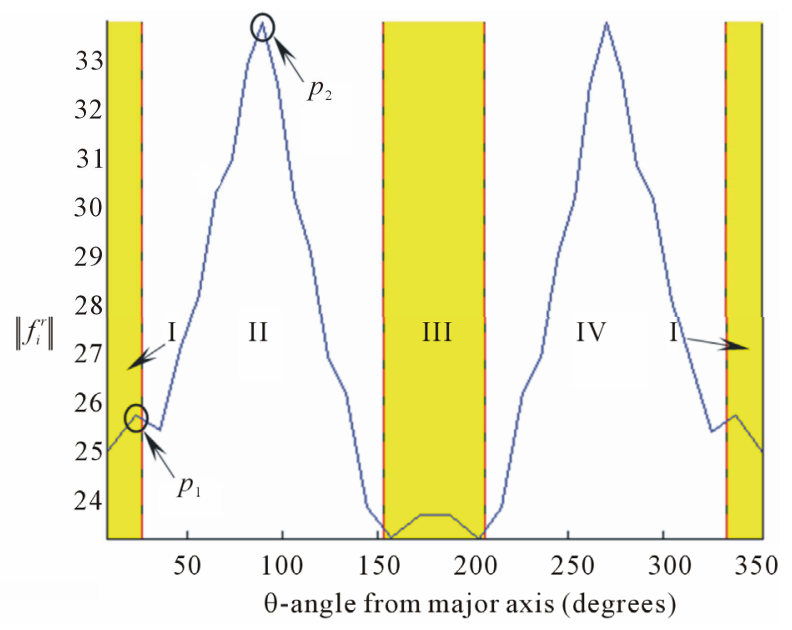

Figure 11. Repulsive force variation along the perimeter of an ellipse.

distribution of these forces is expected to be symmetric with respect to the major (or minor) axis. The slight asymmetry present in this Figure $\mathbf{1 0}$ is due to the selected distribution of the agents (not shown) which is not perfectly symmetric. However, the maximum repulsive forces in regions I and III are very close (as well as those in II and IV), and they are taken as equal. That is, the values at $p_{1}$ and $p_{2}$ are used for the zones II and IV, and I and III respectively.

To accommodate the difference in repulsive force bounds in different regions, we utilize a more conservative strategy using Figure 7 in zones II and IV, and scale the outcome down for zones I and III according to the ratio of the forces at $p_{1}$ and $p_{2}$ (Figure 11). The precise value of this ratio for a given ellipse and radius of interaction is unnecessary and a simple approximation can be used instead. Figure 12 shows the ratio of the force at $p_{2}$ (denoted as $f_{2}^{r}$ ) to the force at $p_{1}$ (i.e., $f_{1}^{r}$ ) for configurations with varying interaction radii and different major and minor axes, with the aspect ratios varying between 0.3 and 1 . This figure was created using sample elliptical configurations and evaluating the maximum of repulsive forces in the different regions. This was performed for 100, 200, 300, 400 and 500 agents, and Figure 12 illustrates the average of these evaluations just to reduce its dependence on the agent count $\mathrm{M}$. Aspect ratios smaller than 0.3 were not considered due to numerical and coding complexities arising in these thin ellipses.

A closer look at Figure 12 reveals an almost constant $f_{1}^{r} / f_{2}^{r}$ ratio, which averages to 0.83 for aspect ratios between 0.3 and 0.1 . This ratio is adopted for the robust control formation here. Bear in mind that we are reduceing an already overly-conservative value from Figure 7, and so the exact ratio for a specific geometry is not necessary to determine a further conservative value for $f_{1}^{r} / f_{2}^{r}$.

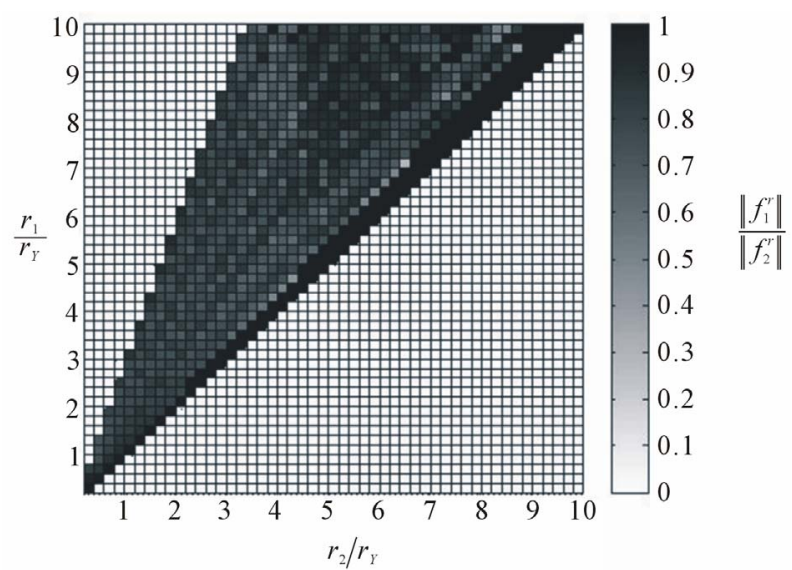

Figure 12. Ratio of force at $p_{2}$ to $p_{1}$ for varying ellipse geometry.

We should state, however, that the impact of the limitation of $0.3<r_{2} / r_{1}<1$ is left for future research. The deployment of this routine for very thin elliptical formations requires different procedures in non isotropic SMC. We also whish to extend the similar needs for target regions that are not elliptical, and further, non convex. The proposed logic which stems from the SMC philosophy fails, as such regions do not have a center of symmetry. Even if they do, the anisotropy can be so strong that it precludes the creation of the equivalent of Figure 12.

Note also that both $K_{1} \mathbf{s}_{i}$ and $K_{2} \cdot \operatorname{sat}\left(\mathbf{s}_{i}, \varepsilon\right) \mathbf{s}_{i} /\left\|\mathbf{s}_{i}\right\|$ terms in (14) are the forces pointing the center of the target ellipse. Because of the sizable boundary layer, the $K_{1} \mathbf{s}_{i}$ term is non-negligible at the border of the region. In order to create the elliptical distribution, the $K_{1}$ term is also scheduled in the four regions (I-IV) based on the proportionality of the $f_{\max }^{r}$ selections for the respective regions as described above.

\section{Case Studies for Elliptical Targets}

Case Study 3, illustrated in Figure 13, uses an elliptical region with major axis $r_{1}=4$, minor axis $r_{2}=2$, and obliqueness $\phi=\pi / 6$. The matrix

$$
\mathbf{T}=\left[\begin{array}{cc}
0.1094 & -0.0812 \\
-0.0812 & 0.2031
\end{array}\right]
$$

contains this information, and the target region again follows a similar desired trajectory (shown by a trace) $\mathbf{x}_{d}=\left[\begin{array}{ll}3 \sin (4 t) & 3 \cos (2 t)\end{array}\right]^{T}$. We use 80 agents, and again add a drag force $\mathbf{f}_{i}^{u}=-(20+5 \sin (2 t)) \dot{\mathbf{x}}_{i} /\left\|\dot{\mathbf{x}}_{i}\right\|$ of which only the upper bound $f_{\max }^{u}=25$ is known to the controller.

After 10 seconds, all the agents are collected inside the region. The area near the end of the major axis is not fully occupied, and agents are somewhat bunched near the middle due to the formation of estimated $f_{\max }^{r}$ which 

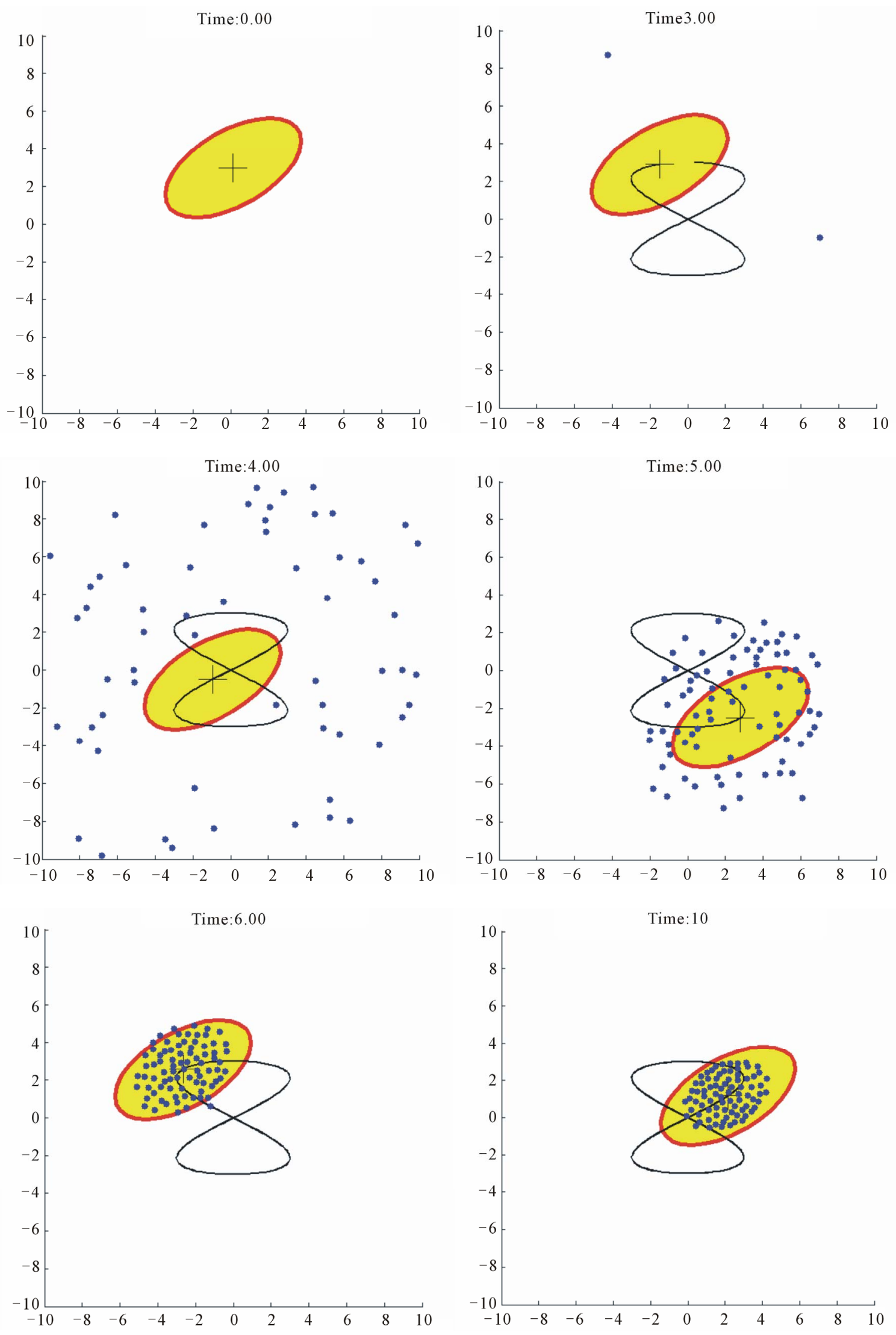

Figure 13. Case study 3: 80 agents tracking a moving elliptical target. 
is over-conservative along the major axis (regions I and III in Figure 10) than it is in the transverse direction (regions II and IV). Nevertheless, the agents are attracted to within the desired target region together with their neighbors.

We again use the quantitative representation of coverage index, $C$, for this case. It is shown in Figure 14, with the marked values at $4,5,6$, and $10 \mathrm{sec}$. Notice the $C(t=10) \approx 0.65$, which represents a somewhat bunched up distribution inside the target region. This is, again, an outcome due to the over conservative $f_{\max }^{r}$ selection along the major axis.

Figures 15 and 16 show the time variations of the sliding function and the control forces respectively. We notice similar dynamics to those in Case study 2: the sliding occurs after approximately 0.8 seconds. The net force at the steady state is again periodic in nature, related to that of region's motion. Same argument can be made here for

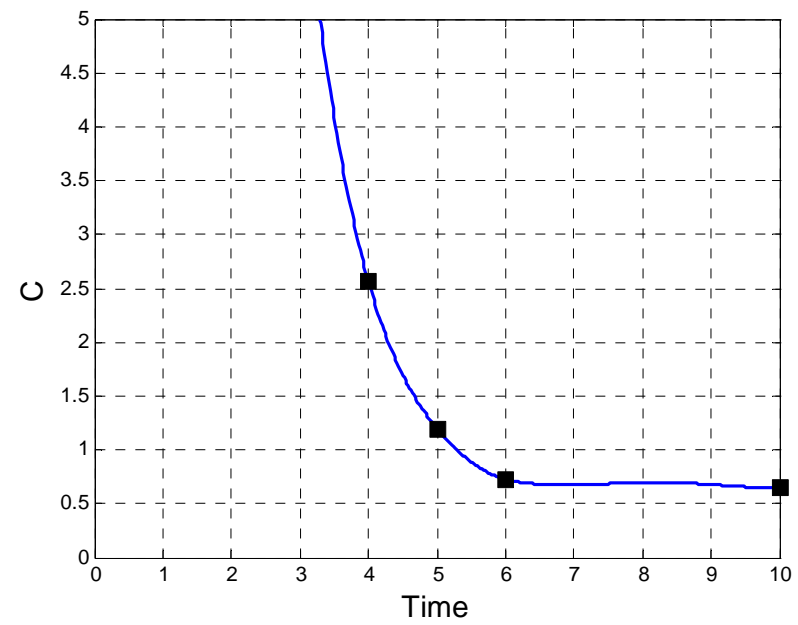

Figure 14. Coverage index variations in Case study 3.
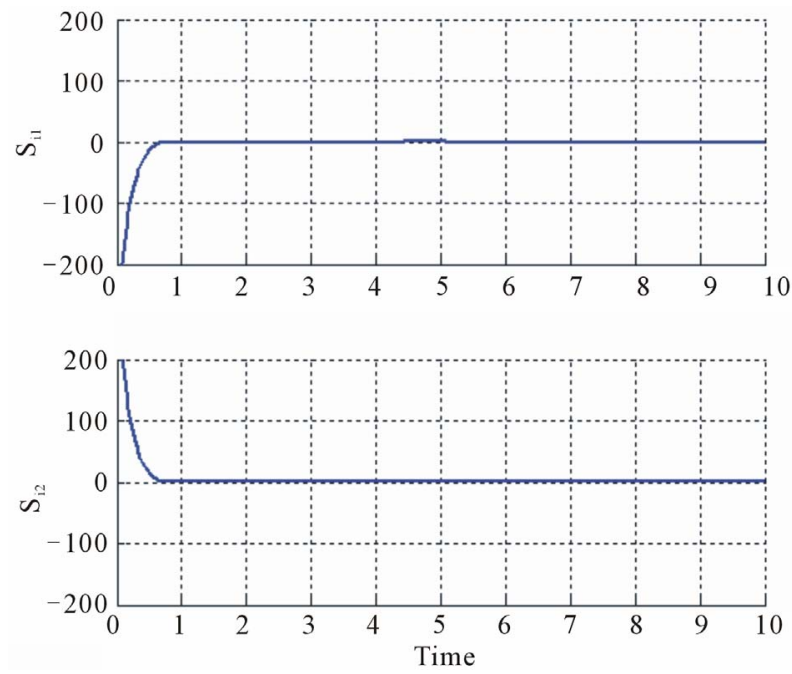

Figure 15. Sliding function of an agent during in Case study 3.
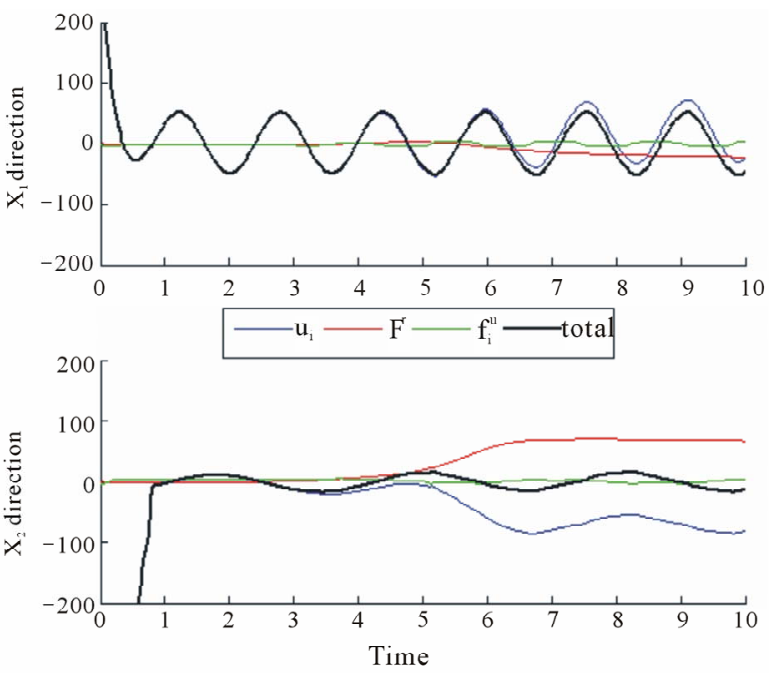

Figure 16. Forces exerted on an agent during in Case study 3.

the fluctuation of the $x_{1}$ and $x_{2}$ directional forces, as we presented over Case study 2.

\section{Conclusions}

In this article we present a decentralized, scalable, sliding mode controller capable of driving individual agents of a swarm into desired circular or elliptical regions, while maintaining a roughly uniform distribution. This controller uses a novel large boundary layer concept and ensures that the agents reach the target region. The outlook of this distribution has direct relation to the form of inter-agent repulsion forces and their a priori known upperbounds. A way of estimating these upperbounds is also presented.

The controller is robust against parametric variations in the model and bounded uncertain forces. It is decentralized, with the agents only knowing the desired target region and the behavior of other agents present in their neighborhood.

Although the assumed distributions were all calculated in a 2-D space, the controller logic is scalable for higher dimensions. This treatment could find applications of swarm aggregation, area coverage, and herding of another swarm.

\section{REFERENCES}

[1] R. Murray, "Recent Research in Cooperative Control of Multi-Vehicle Systems," Journal of Dynamic Systems, Measurement and Control, Vol. 129, No. 5, 2007, pp. 571583. doi:10.1115/1.2766721

[2] K. Warburton and J. Lazarus, "Tendency-Distance Models of Cohesion in Animal Groups," Journal of Theoretical Biology, Vol. 150, No. 4, 1991, pp. 473-488. doi:10.1016/S0022-5193(05)80441-2 
[3] S. Camazine, J. Deneubourg and N. Franks, "Self Organization in Biological Systems," Princeton University Press, Princeton, 2001.

[4] C. Reynolds, "Flocks, Herds and Schools: A Distributed Behavioral Model," Computer Graphics, Vol. 21, No. 4, 1987, pp. 25-34. doi:10.1145/37402.37406

[5] V. Gazi and K. Passino, "Stability Analysis of Swarms," IEEE Transactions on Automatic Control, Vol. 48, No. 4, 2003, pp. 692-697. doi:10.1109/TAC.2003.809765

[6] V. Gazi and K. Passino, "A Class of Attractions/Repulsion Functions for Stable Swarm Aggregations," International Journal of Control, Vol. 77, No. 18, 2004, pp. 1567-1579. doi:10.1080/00207170412331330021

[7] R. Olfati-Saber, "Flocking for Multi-Agent Dynamic Systems: Algorithms and Theory," IEEE Transactions on Automatic Control, Vol. 51, No. 3, 2006, pp. 401-420. doi:10.1109/TAC.2005.864190

[8] H. Su, X. Wang and Z. Lin, "Flocking of Multi-Agents with a Virtual Leader," IEEE Transactions on Automatic Control, Vol. 54, No. 2, 2009, pp. 293-307. doi:10.1109/TAC.2008.2010897

[9] H. Tanner, A. Jadbabaie and G. Pappas, "Flocking in Fixed and Switching Networks," IEEE Transactions on Automatic Control, Vol. 52, No. 5, 2007, pp. 869-868. doi:10.1109/TAC.2007.895948

[10] M. Zavlanos, H. Tanner, A. Jadbabaie and G. Pappas, "Hybrid Control for Connectivity Preserving Flocking," IEEE Transactions on Automatic Control, Vol. 54, No. 12, 2009, pp. 2869-2875. doi:10.1109/TAC.2009.2033750

[11] J. Yao, R. Ordonez and V. Gazi, "Swarm Tracking Using Artificial Potentials and Sliding Mode Control," Journal of Dynamic Systems, Measurement and Control, Vol. 129, No. 5, 2007, pp. 749-754. doi:10.1115/1.2764511
[12] J. Cortes, S. Martinez, T. Karatas and F. Bullo, "Coverage Control for Mobile Sensing Networks," IEEE Transactions on Robotics and Automation, Vol. 20, No. 2, 2004, pp. 243-255. doi:10.1109/TRA.2004.824698

[13] K. Laventall and J. Cortes, "Coverage Control by MultiRobot Networks with Limited-Range Anisotropic Sensory," International Journal of Control, Vol. 82, No. 6, 2009, pp. 1113-1121. doi:10.1080/00207170802471211

[14] F. Bullo, J. Cortes and S. Martinez, "Distributed Control of Robotic Networks," Princeton University Press, Princeton, 2006.

[15] C. Cheah, S. Hou and J.-J. Slotine, "Region-Based Shape Control for a Swarm of Robots," Automatica, Vol. 45, No. 10, 2009, pp. 2406-2411. doi:10.1016/j.automatica.2009.06.026

[16] P. McCullough, M. Bacon, N. Olgac, D. A. Sierra and R. Cepeda-Gomez, "A Lyapunov Treatment of Swarm Coordination under Conflict," Journal of Vibration and Control, Vol. 17, No. 5, 2011, pp. 641-650. doi:10.1177/1077546309360047

[17] V. I. Utkin, "Variable Structure Systems with Sliding Modes," IEEE Transactions on Automatic Control, Vol. AC-22, No. 2, 1977, pp. 212-222. doi:10.1109/TAC.1977.1101446

[18] J.-J. Slotine, "Sliding Controller Design for Non-Linear Systems," International Journal of Control, Vol. 40, No. 2, 1984, pp. 421-434. doi:10.1080/00207178408933284

[19] H. Elmali and N. Olgac, "Sliding Mode Control with Perturbation Estimation (SMCPE): A New Approach," International Journal of Control, Vol. 56, No. 4, 1992, pp. 923-941. doi:10.1080/00207179208934350

[20] J. LaSalle, "Some Extensions of Liapunov's Second Method," IRE Transactions on Circuit Theory, Vol. 7, No. 4, 1960, pp. 520-527. 